\title{
Vectorcardiography for Optimization of Stimulation Intervals in Cardiac Resynchronization Therapy
}

\author{
Caroline J. M. van Deursen • Liliane Wecke • Wouter M. van Everdingen • \\ Marcus Ståhlberg • Michel H. G. Janssen • Frieder Braunschweig • \\ Lennart Bergfeldt • Harry J. G. M. Crijns • Kevin Vernooy • Frits W. Prinzen
}

Received: 20 November 2014 / Accepted: 12 February 2015 / Published online: 6 March 2015

(C) The Author(s) 2015. This article is published with open access at SpringerLink.com

\begin{abstract}
Current optimization of atrioventricular (AV) and interventricular (VV) intervals in cardiac resynchronization therapy (CRT) is time consuming and subject to noise. We aimed to prove the principle that the best hemodynamic effect of CRT is achieved by cancelation of opposing electrical forces, detectable from the QRS morphology in the 3D vectorcardiogram (VCG). Different degrees of left (LV) and right ventricular $(\mathrm{RV})$ pre-excitation were induced, using variation in $\mathrm{AV}$ intervals during $\mathrm{LV}$ pacing in 20 patients with left bundle branch block (LBBB) and variation in VV intervals during biventricular pacing in 18 patients with complete AV block or atrial fibrillation. The smallest QRS vector area identified stimulation intervals with minimal systolic stretch (median difference [IQR $] 20 \mathrm{~ms}[-20,20 \mathrm{~ms}]$ and maximal hemodynamic response (10 ms [-20, $40 \mathrm{~ms}])$. Reliability of VCG
\end{abstract}

Associate Editor Craig Stolen oversaw the review of this article

Caroline J. M. van Deursen contributed equally to this work.

C. J. M. van Deursen • W. M. van Everdingen · F. W. Prinzen Departments of Physiology, Cardiovascular Research Institute

Maastricht, Maastricht, The Netherlands

C. J. M. van Deursen • M. H. G. Janssen • H. J. G. M. Crijns •

K. Vernooy

Departments Cardiology, Cardiovascular Research Institute

Maastricht, Maastricht, The Netherlands

L. Wecke $\cdot$ M. Ståhlberg $\cdot$ F. Braunschweig

Karolinska Institutet, Department of Cardiology, Karolinska

University Hospital, Stockholm, Sweden

\section{Bergfeldt}

Sahlgrenska Academy, Department of Molecular and Clinical

Medicine/Cardiology, University of Gothenburg,

Gothenburg, Sweden

F. W. Prinzen $(\square)$

P.O. Box 616, 6200, MD Maastricht, The Netherlands

e-mail: frits.prinzen@maastrichtuniversity.nl measurements was superior to hemodynamic measurements. This study proves the principle that VCG analysis may allow easy and reliable optimization of stimulation intervals in CRT patients.

Keywords Cardiac resynchronization therapy - Biventricular pacing · Vectorcardiography · Electrocardiography ·

Atrioventricular timing $\cdot$ Interventricular timing

\section{Introduction}

In cardiac resynchronization therapy (CRT), the time intervals between electrical stimulation of the right atrium and ventricles (atrioventricular (AV) interval) and between the right and left ventricle (interventricular (VV) interval) determine left ventricular (LV) filling characteristics and the degree of ventricular resynchronization. Multiple techniques are used for optimization of the AV and VV intervals, such as echocardiography (LV outflow tract velocity time integral, mitral inflow patterns), invasive hemodynamics ( $\mathrm{LV} d P / d t_{\max }$ ), and finger blood pressure measurements and electrogram (EGM)-based methods. Acute hemodynamic improvements by up to $\sim 15 \%$ for $\mathrm{AV}$ and $\mathrm{VV}$ optimization compared to nominal settings have been described [1-4]. However, favorable long-term effects in large clinical trials have not been observed [5-8]. One of the reasons for the absent evidence of long-term benefit might be that the parameters used for optimization have a high measurement variability, leading to a low "signal to noise ratio" $[9,10]$. This seems even the case for invasively measured LV $d P / d t_{\max }$, often regarded as the gold standard of hemodynamic response [2]. The lack of evidence for longterm benefit of optimization and the time-consuming nature of the methods used to optimize CRT make it understandable that a vast majority of implanting physicians leave CRT device settings at their nominal values ("out-ofthe-box") [11]. 
Several algorithms have been introduced to perform CRT optimization in an automated device-based manner. Some algorithms are based on baseline intracardiac EGMs, predicting maximal resynchronization [5, 12]; others use special sensors (accelerometer for determination of peak endocardial acceleration) [13], but clinical benefits have only been demonstrated for the adaptive CRT algorithm in patients where synchronized LV pacing was performed [14].

Vectorcardiography (VCG) contains 3D information of electrical forces within the heart and thus may provide a valuable description of the degree of resynchronization during LV or biventricular $(\mathrm{BiV})$ pacing. We recently showed in ischemic and nonischemic failing canine hearts with left bundle branch block (LBBB) that VCG reflects electrical interventricular dyssynchrony and is a reliable and reproducible tool for $\mathrm{AV}$ and VV optimizations [15].

The present study was designed to prove the principle of using 3D VCG for optimization of AV and VV intervals in CRT recipients. We hypothesized that optimal AV and VV intervals are predicted by the smallest QRS vector area $\left(\mathrm{QRSV}_{\mathrm{AREA}}\right)$, because it expresses the highest degree of opposition of electrical forces from two directions ("cancelation") [16], implying the best electrical resynchronization. VCG predictions of optimal AV and VV intervals were assessed in relation to those determined by noninvasive hemodynamic measurements and strain patterns.

\section{Methods}

\section{Patient selection}

The study population consisted of 38 patients, implanted with a CRT device according to current guidelines, with either sinus rhythm and LBBB ( $n=20$; AV group) or complete AV block/atrial fibrillation with a slow ventricular rate $(n=18$; VV group). Measurements were performed at least 3 months after CRT implantation. In the AV group, we investigated the full range of electrical dyssynchrony by studying activation sequences caused by intrinsic (LBBB) conduction and by LV pacing in combination with various degrees of fusion with intrinsic conduction. Previous studies have shown that by using LV pacing at various AV intervals, all degrees of dyssynchrony can be studied that are achieved by BiV pacing at various $\mathrm{AV}$ and $\mathrm{VV}$ intervals [17-19]. The VV group was used to investigate the effect of right ventricular (RV) and LV pacing without fusion with intrinsic conduction.

The study was performed according to the principles of the Declaration of Helsinki and approved by the ethics committee of Maastricht University Hospital and the regional ethical review board in Stockholm. All participants gave fully informed written consent prior to investigation.

\section{Pacing Protocol}

In the AV group, single chamber LV pacing was performed during overdrive atrial pacing $(80 \mathrm{bpm})$ at $\mathrm{AV}$ intervals ranging from $50 \mathrm{~ms}$ until intrinsic conduction (ventricular noncapture) with steps of 20-25 ms. AAI pacing at $80 \mathrm{bpm}$ was used as baseline. In the VV group, $\mathrm{BiV}$ pacing was performed at a rate of $80 \mathrm{bpm}$ with $\mathrm{VV}$ intervals ranging from $80 \mathrm{~ms} \mathrm{LV}$ pre-excitation (negative values) to $80 \mathrm{~ms} \mathrm{RV}$ preexcitation (positive values) in $20 \mathrm{~ms}$ steps, using simultaneous $\mathrm{BiV}$ pacing (VV interval of $\sim 0 \mathrm{~ms}$ ) as baseline. In the patients with complete $\mathrm{AV}$ block $(n=12)$, the $\mathrm{AV}$ interval was kept constant with the time from paced right atrium to the first paced ventricle (either right or left sided) being $120 \mathrm{~ms}$. The pacing protocols were alternatingly executed in a top-down (starting with short AV delays and maximal LV pre-excitation) or a bottom-up order to minimize potential bias.

Vectorcardiographic and Electrocardiographic Measurements

VCG was performed using eight electrodes positioned according to the modified Frank orthogonal lead system $(X, Y$, and $Z$; Coronet II System, Ortivus AB, Danderyd, Sweden) with patients at rest and in supine position. With each pacing configuration, recordings were made at a sampling frequency of $500 \mathrm{~Hz}$ for $3 \mathrm{~min}$ and signals were averaged over half a minute. The VCGs were analyzed offline using customized software [20]. The maximum distance between the origin $(0,0,0)$ and a point on the $3 \mathrm{D} Q \mathrm{QRS}$ vector loop was represented by the maximal QRS vector amplitude $\left(\mathrm{QRSV}_{\mathrm{AMPL}}(\mathrm{mV})\right)$. The direction of the maximal QRS vector in space was determined using the angle in the transversal plane and the angle in craniocaudal direction. The $\mathrm{QRS}$ vector area $\left(\mathrm{QRSV}_{\mathrm{AREA}}\right.$ $(\mu \mathrm{Vs}))$ was assessed as the "3D area" between the curve and the baseline from the beginning to the end of the QRS complex in $X, Y$, and $Z$, and calculated as $\left(\mathrm{QRS} x^{2}+\right.$ $\left.\mathrm{QRS} y^{2}+\mathrm{QRS}^{2}\right)^{1 / 2}$.

Because VCG measurements are not standard, we also investigated the possibility to use the standard 12-lead ECG for optimization of CRT. To this purpose, the ECG was recorded for each pacing configuration. Analysis was performed on the ECG lead that showed the largest change in QRS amplitude between LBBB or RV pacing and LV pacing, thereby reflecting the largest projection of the 3D QRS vector loop. For each patient, this lead was defined as lead $\mathrm{MAX}_{\text {. }}$. Based on previous studies in canine hearts [15], we predicted the optimal AV and VV intervals from the QRS amplitude in lead $\mathrm{MAX}_{\mathrm{X}}$ ( $\mathrm{QRSE}_{\mathrm{AMPL}}$ ), tracing a value closest to that halfway in 
between fully captured LV pacing and LBBB or RV pacing $\left(\triangle \mathrm{QRSE}_{\mathrm{AMPL} H W}\right)$. QRSE $\mathrm{AMPL}_{\mathrm{AM}}$ was measured manually as the net deflection of the QRS complex, defining $\mathrm{rS}$ or QS morphologies as negative and R or Rs morphologies as positive.

\section{Finger Blood Pressure Measurements}

Finger blood pressure measurements were acquired using a plethysmographic approach (Nexfin, BMEYE B.V., Amsterdam, The Netherlands). Systolic blood pressure (SBP) and stroke volume (SV) were derived from the continuous signal. This algorithm has previously been validated for optimization of CRT settings [21]. Each pacing configuration was directly compared to the baseline situation during two transitions (from baseline to a stimulation interval and back). The ten beats before and after each transition were selected and averaged using customized Matlab software (MathWorks, Natick, MA, USA) [21].

\section{Echocardiographic Measurements}

After the simultaneous VCG and finger blood pressure measurements for all pacing configurations, transthoracic echocardiographic recordings were made using an $\mathrm{iE} 33$ system with S5-1 transducer (Philips Medical Systems, Best, The Netherlands). LV outflow tract velocity time integral $\left(\mathrm{VTI}_{\mathrm{LVOT}}\right)$ was calculated offline for six consecutive beats. Pulsed wave Doppler echocardiography was used to measure interventricular mechanical delay (IVMD) as the difference between time to onset of pulmonary and aortic ejection (a negative IVMD indicating earlier aortic ejection). Apical four-chamber views were obtained with high frame rates $(60-90 \mathrm{~Hz})$ during breath-hold at end-expiration for three consecutive beats. In these images, septal strain was determined offline in the middle $50 \%$ of the septal segment using speckle tracking software (QLAB version 8.1, Philips Medical Systems, Bothell, WA, USA). From the septal strain signals, two indices were calculated using customized Matlab software: septal systolic pre-stretch (SPS), defined as early systolic septal stretch and representing late septal activation (septal stretching due to early unopposed contralateral LV free wall contraction), and septal systolic rebound stretch (SRS), defined as systolic stretch following initial shortening and representing early septal activation (septal stretching due to late vigorous contralateral LV free wall contraction) [22]. Such stretch during the systolic phase diminishes effective pumping, since the stretch "absorbs" the shortening of other ventricular wall segments. Consequently, the lowest amount of stretch during systole (SPS + SRS) was considered to represent the most physiological state.
Determination of the Optimal Hemodynamic AV or VV Interval

The hemodynamic effect of changing the AV or VV interval was defined as the relative change from baseline for the combination of $\mathrm{VTI}_{\mathrm{LVOT}}$ and finger blood pressure measurements; $\Delta$ HEMO $(\%)=0.5 \times\left(\Delta \mathrm{VTI}_{\mathrm{LVOT}}+0.5 \times(\Delta \mathrm{SBP}+\Delta \mathrm{SV})\right)$. Combining the data from the independently performed finger blood pressure and $\mathrm{VTI}_{\mathrm{LVOT}}$ measurements can be expected to improve the accuracy of the measurement of the hemodynamic effect [9]. The AV or VV interval with the largest $\triangle \mathrm{HEMO}$ was considered the optimal interval. All assessors were blinded to the results from the other methods.

\section{Statistical Analysis}

Statistical analyses were performed using IBM SPSS Statistics software version 20 (SPSS Inc., Chicago, IL). Continuous variables were presented as mean $\pm \mathrm{SD}$ or as median and interquartile range (IQR; 25th-75th percentile) in case of nonnormal distribution, categorical variables as number (percentage). Mutual linear correlations between different parameters at the various $\mathrm{AV}$ and VV intervals were evaluated by Pearson's correlation coefficient. Agreement between the various predictions of optimal $\mathrm{AV}$ and $\mathrm{VV}$ intervals was evaluated with a BlandAltman analysis, containing the mean difference (bias) and the limits of agreement (defined as mean $\pm 1.96^{*} \mathrm{SD}$ ) [23]. The goodness of fit of raw data points at the various $\mathrm{AV}$ and $\mathrm{VV}$ intervals with a fitted sixth order polynomial curve (expressed as $R^{2}$ ) was used as an indicator of reliability of the measurements (less noise in a measurement will produce a better fit).

\section{Results}

Clinical characteristics are presented in Table 1 and are fairly representative for a CRT population.

Magnitude and Direction of the QRS Vector During LBBB, RV Pacing, and LV Pacing

During intrinsic conduction in the AV group (LBBB), the QRS vector pointed toward the back and left side of the patient with the highest amplitude in the transversal plane (Fig. 1). In contrast, during RV pacing in the VV group, the vector pointed more in the craniocaudal direction (upward). LV pacing with a short $\mathrm{AV}$ interval reversed the vector toward the front and right side of the patient. Changes in $\mathrm{QRSV}_{\mathrm{AMPL}}$ and its orientation in the frontal and transversal plane are shown for representative patients of the $\mathrm{AV}$ and $\mathrm{VV}$ groups during all stimulation intervals (Fig. 1, lower panels). 
Table 1 Baseline characteristics

CRT response was defined as a $\geq 5 \%$ point improvement in $\mathrm{LVEF}$ after 3-6 months of CRT determined by echocardiography according to the biplane method of disks (modified Simpson)

$H F$ heart failure, $L B B B$ left bundle branch block, $R V$ right ventricle, $A V B$ complete $\mathrm{AV}$ block, $L V E F$ left ventricular ejection fraction, LVESV left ventricular end systolic volume, NYHA New York Heart

Association functional class, $C R T$ cardiac resynchronization therapy, $L V$ left ventricle, $A C E$ angiotensin-converting enzyme, $A R B$ angiotensin II type 1 receptor blocker

\begin{tabular}{|c|c|c|c|}
\hline Patient characteristics, $n(\%)($ mean \pm SD $)$ & AV group $(n=20)$ & VV group $(n=18)$ & Total $(n=38)$ \\
\hline Age (years) & {$[64.2 \pm 8.2]$} & {$[68.8 \pm 8.9]$} & {$[66.3 \pm 8.7]$} \\
\hline Male gender & $20(100 \%)$ & $14(78 \%)$ & $34(89 \%)$ \\
\hline Ischemic HF etiology & $8(40 \%)$ & $10(56 \%)$ & $18(47 \%)$ \\
\hline \multicolumn{4}{|l|}{ Pre-CRT values } \\
\hline QRS duration (ms) & {$[167 \pm 20]$} & {$[177 \pm 19]$} & {$[172 \pm 20]$} \\
\hline LBBB morphology & $19(95 \%)$ & $4(22 \%)$ & $23(61 \%)$ \\
\hline RV pacing/AVB & $0(0 \%)$ & $13(72 \%)$ & $13(34 \%)$ \\
\hline LVEF (\%) & {$[25 \pm 7]$} & {$[26 \pm 10]$} & {$[25 \pm 9]$} \\
\hline LVESV (ml) & {$[187 \pm 71]$} & {$[154 \pm 62]$} & {$[171 \pm 68]$} \\
\hline CRT responders & $15(75 \%)$ & $10(56 \%)$ & $25(66 \%)$ \\
\hline \multicolumn{4}{|l|}{ RV lead position } \\
\hline RV outflow tract & $5(25 \%)$ & $1(6 \%)$ & $6(16 \%)$ \\
\hline Septum & $0(0 \%)$ & $1(6 \%)$ & $1(3 \%)$ \\
\hline Apex & $15(75 \%)$ & $16(89 \%)$ & $31(82 \%)$ \\
\hline \multicolumn{4}{|l|}{ LV lead position } \\
\hline Anterior & $0(0 \%)$ & $1(6 \%)$ & $1(3 \%)$ \\
\hline Anterolateral & $8(40 \%)$ & $5(28 \%)$ & $13(34 \%)$ \\
\hline Lateral & $3(15 \%)$ & $1(6 \%)$ & $4(11 \%)$ \\
\hline Inferolateral & $8(40 \%)$ & $8(44 \%)$ & $16(42 \%)$ \\
\hline Inferior & $1(5 \%)$ & $3(17 \%)$ & $4(11 \%)$ \\
\hline \multicolumn{4}{|l|}{ Medication } \\
\hline$\beta$-Blocker & $20(100 \%)$ & $17(94 \%)$ & $37(97 \%)$ \\
\hline ACE-inhibitor/ARB & $19(95 \%)$ & $18(100 \%)$ & $37(97 \%)$ \\
\hline Loop diuretics & $10(50 \%)$ & $12(67 \%)$ & $22(58 \%)$ \\
\hline Aldosterone antagonist & $9(45 \%)$ & $10(56 \%)$ & $19(50 \%)$ \\
\hline Amiodarone & $1(5 \%)$ & $1(6 \%)$ & $2(5 \%)$ \\
\hline
\end{tabular}

Effect of Different Degrees of LV and RV Pre-Excitation on Electrical, Mechanical, and Hemodynamic Parameters

In Fig. 2, an overview of all electrical (ECG and VCG), mechanical (IVMD, SPS, and SRS), and hemodynamic ( $\mathrm{VTI}_{\mathrm{LVOT}}$ and SBP) parameters is presented as measured at different stimulation intervals for a representative patient of the AV group. When increasing the AV interval from 50 to $160 \mathrm{~ms}$, the morphology of the ECG and VCG gradually changed, characterized by a decrease of QRS amplitudes and area. By further prolongation of the AV interval, these variables increased again, yet showing an opposite direction. IVMD started with earlier aortic ejection when applying LV pre-excitation, gradually becoming more synchronous and ending with earlier pulmonary ejection when loosing LV capture. With short AV intervals, there was considerable SPS, indicating passive early systolic septal stretch caused by early LV free wall activation, which gradually decreased and disappeared with longer AV intervals. SRS was absent during LV pre-excitation and increased with prolonging $\mathrm{AV}$ intervals. $\mathrm{VTI}_{\mathrm{LVOT}}$ and $\mathrm{SBP}$ increased when increasing AV interval from 50 to $160 \mathrm{~ms}$ and subsequently decreased again.

Prediction of the Optimal AV and VV Interval with Vectorcardiography

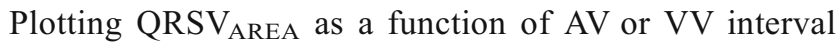
showed a parabolic curve with a distinct minimum in the middle (Fig. 3). At the stimulation interval with smallest $\mathrm{QRSV}_{\text {AREA }}$, systolic stretch was lowest and hemodynamic improvement was largest, also for the entire group (Fig. 4, Table 2). Combining data from the AV group and VV group, the smallest QRS vector area identified stimulation intervals with minimal systolic stretch (median difference $20 \mathrm{~ms}$ [IQR -20, $20 \mathrm{~ms}]$ ) and maximal hemodynamic response (10 ms [-20, $40 \mathrm{~ms}])$.

Prediction of the Optimal AV and VV Interval with Electrocardiography

We reasoned that the effect of resynchronization is best appreciated and most reliably measured in the ECG lead showing 
Fig. 1 QRS vector amplitude and direction for the different pacing configurations. Upper row: QRS vectors of all patients for intrinsic conduction (LBBB; hyphen; $n=20$ ), RV pacing (diamond; $n=18$ ), and LV pacing (plus sign; $n=38$ ) in the frontal (left) and transversal (right) plane. Middle row: QRS vector amplitude $\left(\mathrm{QRSV}_{\mathrm{AMPL}}\right)$ for a representative patient of the AV group during different $\mathrm{AV}$ intervals with indication of the halfway (HW) value of $\mathrm{QRSV}_{\mathrm{AMPL}}$ in between LV pacing and LBBB. Lower row: $\mathrm{QRSV}_{\mathrm{AMPL}}$ for a representative patient of the $\mathrm{VV}$ group during different $\mathrm{VV}$ intervals with indication of the HW value in between LV and RV pacing. The amplitude of the vector is indicated by the distance of a data point from the center, whereas the angle in a plane is indicated by its position in the $X-Y$ and $X-Z$ plane. Concentric circles indicate $1 \mathrm{mV}$ (upper row) and $0.67 \mathrm{mV}$ (middle and lower rows) amplitude units, respectively

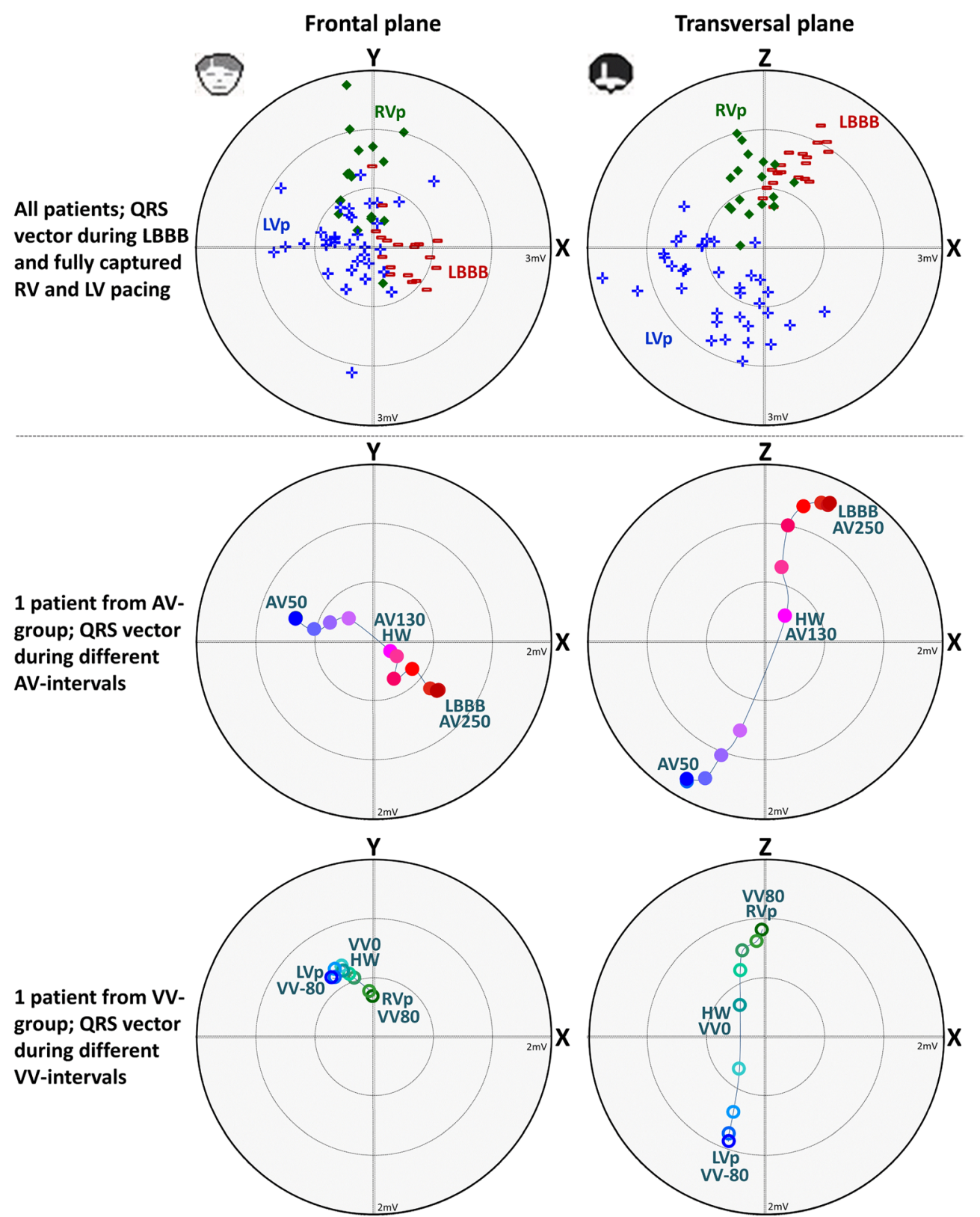

the largest changes in voltage $\left(\right.$ lead $\left._{\text {MAX }}\right)$. This lead MAX $_{\text {was }}$ predominantly found to be lead $\mathrm{V}_{2}$, especially in the $\mathrm{AV}$ group (Fig. 5). The optimal AV and VV interval, predicted from the setting that results in a $\mathrm{QRSE}_{\mathrm{AMPL}}$ closest to the value halfway between LV pacing and LBBB or RV pacing (lowest $\left.\triangle \mathrm{QRSE}_{\mathrm{AMPL}} \mathrm{HW}\right)$ provided a prediction of the interval with lowest systolic stretch and highest $\triangle \mathrm{HEMO}$ that was compa-

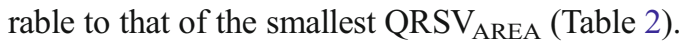

The Hemodynamic Effect of Stimulation Interval Optimization

Compared to a nominal AV interval of $120 \mathrm{~ms}, \triangle \mathrm{HEMO}$ increased by $2.3 \%$ [IQR $0.7-7.3 \%$ ] points at the best $\mathrm{AV}$ interval, with an increase of more than $5 \%$ occurring in $35 \%$ of patients. Similarly, compared to simultaneous BiV pacing, sequential pacing caused a $2.3 \%$ [IQR $0.5-4.3 \%$ ] point increase in $\triangle \mathrm{HEMO}$ with an increase of more than $5 \%$ in $22 \%$ of patients.

Reliability of the Vectorcardiographic Measurements

The goodness of fit of raw data points with a fitted sixth order polynomial curve at various $\mathrm{AV}$ and $\mathrm{VV}$ intervals was highest for $\mathrm{QRSV}_{\mathrm{AREA}}, \mathrm{QRSV}_{\mathrm{AMPL}}$, and QRSE $\mathrm{AMPL}_{\mathrm{AM}}\left(R^{2}=0.99 ; 0.98\right.$; $1.00)$, good for IVMD, SPS, and SRS $\left(R^{2}=0.98 ; 0.97 ; 0.96\right)$, and lowest for $\triangle$ HEMO $\left(R^{2}=0.87\right)$. The high $R^{2}$ value for the electrical parameters indicates minor noise in these measurements, producing a good reliability. 
Fig. 2 Overview of the electrical, mechanical, and hemodynamic parameters at different $\mathrm{AV}$ intervals. A representative example of electrical, mechanical, and hemodynamic parameters while increasing the AV interval during LV pacing. From top to bottom: $\mathrm{ECG}$ in lead $\mathrm{V}_{2}$ (note that the value halfway in between $\mathrm{LV}$ pacing and LBBB is $(-1.9+2.2)$ / $2=0.15)$, VCG in the transversal plane, pulsed wave Doppler signals over the pulmonary and aortic valve with values of the interventricular mechanical delay (IVMD), septal strain curves with values of systolic pre-stretch (SPS) and systolic rebound stretch (SRS; the interval within the vertical lines represents ystole), velocity time integral over the LV outflow tract (VTI $\mathrm{LVOT}_{\text {), finger blood pressure }}$ (FBP) measurements just before and after the transition from intrinsic conduction to a specific $\mathrm{AV}$ interval (for further explanation see text in "Methods")

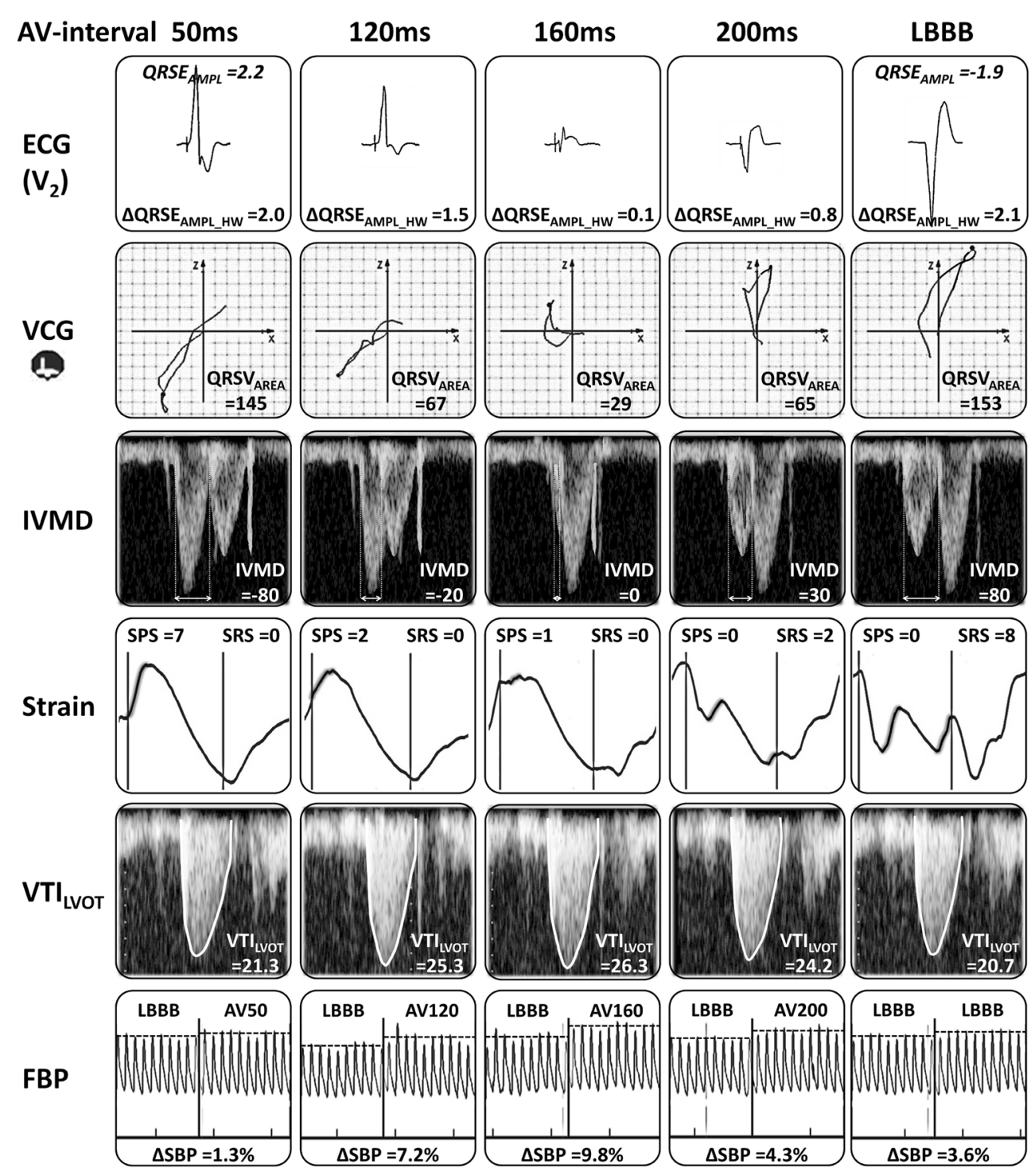

\section{Discussion}

This small study in CRT patients proves the principle that electrical vectorial forces, derived from 3D VCG or the 12lead ECG, can predict the AV and VV intervals that lead to optimal cardiac mechanical and hemodynamic function. This signifies a strong potential of these electrical measurements for routine optimization of the AV and VV intervals in CRT, especially since the method is easy to perform and has a low measuring variability.

Vectorcardiography as an AV and VV Optimization Tool

While most methods for CRT optimization are based on cardiac mechanic or hemodynamic measurements, some studies show that also QRS duration can be used to this purpose, the narrowest QRS complex predicting the largest hemodynamic benefit $[19,24]$. It is plausible that indicators of QRS morphology, like QRS area, are more sensitive and robust predictors of good resynchronization than QRS duration, because the former are based on the entire QRS complex in all leads. This is reminiscent of the better prediction of CRT response by QRS morphology (i.e., LBBB morphology) than by QRS duration alone [25].

Individual determination of maximal cancelation of electrical forces seems important in the light of observations made by noninvasive electrical mapping (ECG imaging), showing that lines of block may appear or disappear during certain pacing configuration in some patients $[26,27]$. The latter observations also indicate why measured markers of electrical dyssynchrony may be preferred over the ones that are predicted based on EGMs measured during intrinsic rhythm as used by several commercial optimization algorithms $[5,12,14]$.

The observation that the VCG approach worked equally well in LBBB and AV block patients indicates that intrinsic 
Fig. 3 Change in electrical, mechanical, and hemodynamic parameters during different stimulation intervals. Presented are $Q R S$ vector area $\left(\right.$ QRSV $\left._{\text {AREA }}\right)$ and interventricular mechanical delay $\left(\Delta \mathrm{IVMD}_{\mathrm{HW}}\right)$ with their values halfway in between those during LV and RV pre-excitation, total septal systolic stretch (SPS+ SRS), and the hemodynamic increase relative to baseline LBBB $(\triangle H E M O)$ as a function of the paced AV interval (left) and VV interval (right) in two representative patients. Note that the stimulation interval with smallest QRSV AREA $_{\text {corresponds }}$ closely with that of lowest $\triangle \mathrm{IVMD}_{\mathrm{HW}}$ and SPS $+\mathrm{SRS}$, and highest $\triangle$ HEMO

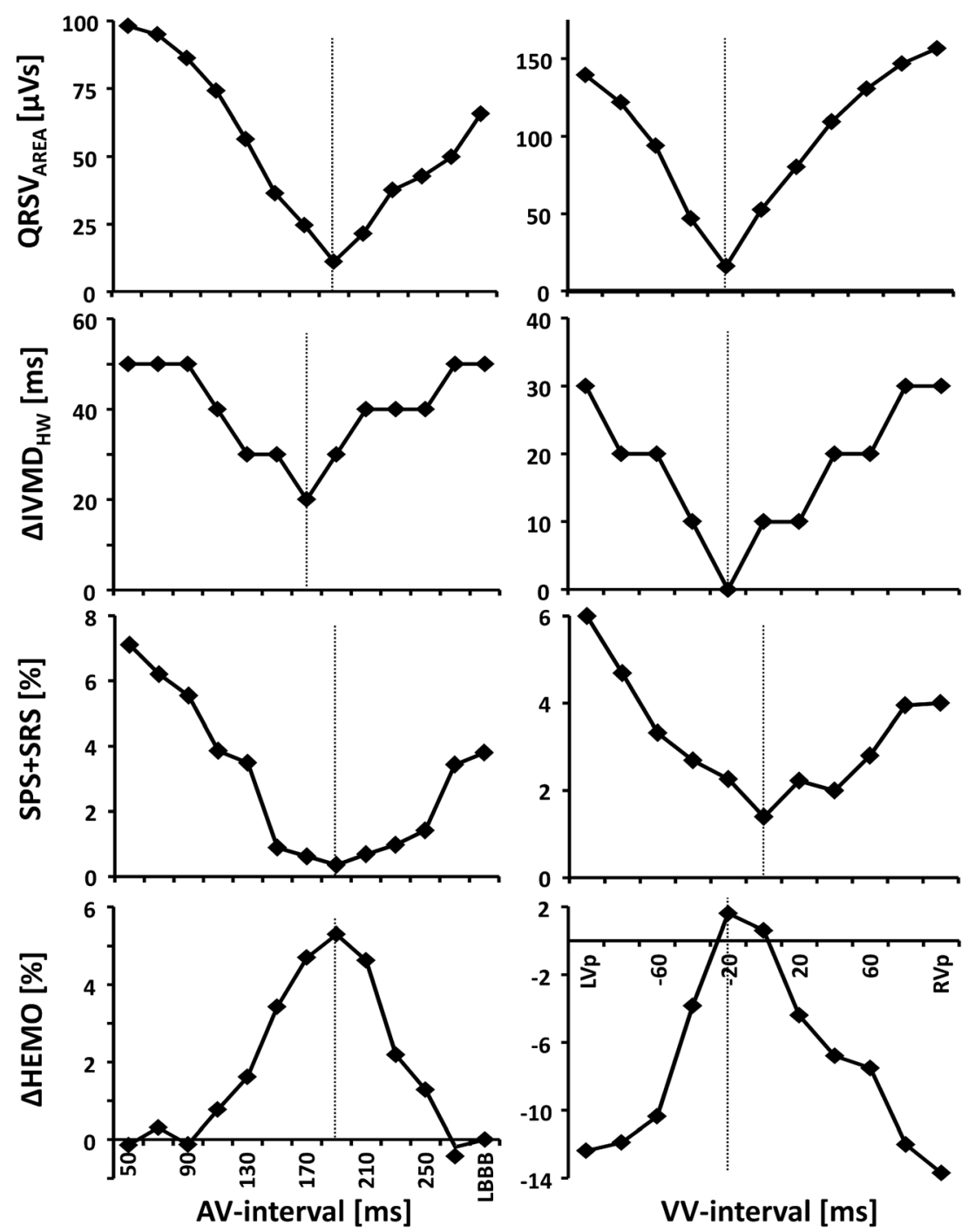

and paced LBBB activation follow similar rules for resynchronization.

Values of the optimal AV interval in the present small study (120-220 ms) appear longer than those observed in other studies [5]. However, it should be kept in mind that in the presently investigated optimization during single site LV pacing, optimal resynchronization requires fusion with the intrinsic activation wavefront.

The use of an AV (and VV) optimization tool that solely focuses on electrical optimization ("resynchronization") seems to ignore the effect of timing on filling of the ventricles. However, both animal and human studies demonstrate that in the range of $\mathrm{AV}$ intervals used for CRT, the effect of the $\mathrm{AV}$ interval on filling is small $[28,29]$. Nevertheless, patients with a restrictive filling pattern and high LV filling pressures prior to CRT may depend more on optimal filling [30].

VCG can easily be applied in clinical practice. In the present study, we used a dedicated 3D VCG system. However, most commercially available ECG machines have algorithms to construct VCGs using the inverse Dower or Kors' regression transformation $[31,32]$. This approach may provide additional information on top of reading the 12 leads (like automatic calculated QRS area), but the accuracy of the inverse transformations has yet to be demonstrated for CRT patients.

\section{Electrocardiography as an AV and VV Optimization Tool}

Clinical application is even easier when using the 12-lead $\mathrm{ECG}$, in particular the lead that shows the largest change in amplitude between LV pacing and LBBB or RV pacing

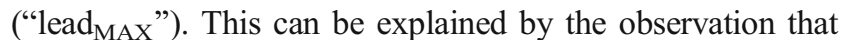
this lead (most commonly $\mathrm{V}_{2}-\mathrm{V}_{3}$ ) is the strongest determinant of the direction and amplitude of the entire 3D VCG (mostly antero-posterior and apico-basal forces in these patients with LBBB and RV pacing, respectively). While VCG may provide a more comprehensive image, "lead $\mathrm{MAX}_{\mathrm{X}}$ " may be easiest to use in clinical practice. 
Fig. 4 Agreement between VCG, mechanical, and hemodynamic predicted stimulation intervals. Correlation between the AV (upper row) and $\mathrm{VV}$ (lower row) intervals with smallest QRSV $V_{\text {AREA }}$ compared to that with lowest systolic stretch (left) and with highest $\triangle \mathrm{HEMO}$ $($ right). Shown is the line of identity $(X=Y)$ and the range within $20 \mathrm{~ms}$ of this line. The area of the dots indicates the number of observations at that specific value; clustering of data points are due to stepwise increase of $\mathrm{AV}$ and VV intervals
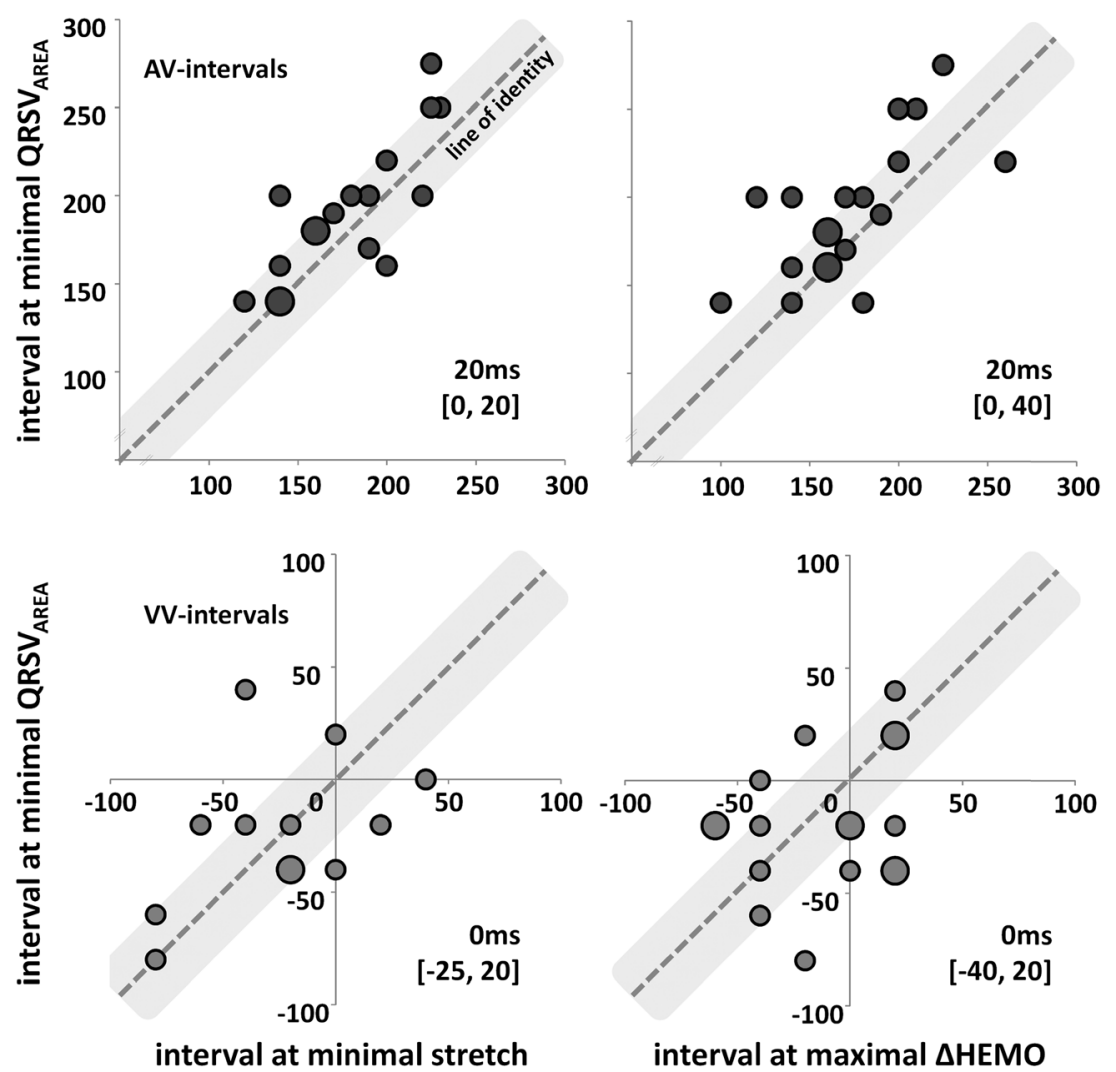

interval at maximal $\triangle \mathrm{HEMO}$
Some physicians use the setting with the smallest QRS duration as a practical optimization method. Recently, this approach was shown to improve acute hemodynamic response compared to nominal AV and VV programming in CRT patients [19]. In our previous animal study, QRS duration appeared to be a worse predictor of the optimal AV and VV interval than the QRS vector amplitude halfway in between LV and RV pacing [15]. An explanation can be that although QRS duration reflects total ventricular activation time, it correlates only poorly with electrical and mechanical interventricular dyssynchrony [15, 28, 33]. Moreover, measuring the QRS

Table 2 Agreement between $\mathrm{QRSV}_{\mathrm{AREA}}$ and $\triangle \mathrm{QRSE}_{\mathrm{AMPL}} \mathrm{HW}$ with systolic stretch and hemodynamic function for prediction of optimal CRT settings

\begin{tabular}{lcc}
\hline Prediction: Bias \pm LoA & lowest $\mathrm{QRSV}_{\text {AREA }}$ & lowest $\Delta \mathrm{QRSE}_{\mathrm{AMPL} \_\mathrm{HW}}$ \\
\hline Lowest systolic stretch & & \\
AV-intervals (ms) & $13 \pm 48$ & $-2 \pm 47$ \\
VV-intervals (ms) & $2 \pm 72$ & $-17 \pm 70$ \\
total (ms) & $8 \pm 59$ & $-8 \pm 58$ \\
Highest $\Delta$ HEMO & & \\
AV intervals (ms) & $19 \pm 60$ & $7 \pm 56$ \\
VV intervals (ms) & $-7 \pm 72$ & $-26 \pm 65$ \\
Total (ms) & $7 \pm 70$ & $-8 \pm 68$ \\
\hline
\end{tabular}

Presented are bias with limits of agreement (LoA); defined as mean difference $\pm 1.96^{*} \mathrm{SD}$ duration is a precise task which requires at least an ECG paper speed of $50 \mathrm{~mm} / \mathrm{s}$ and measurement with digital cursors [19]. Instead, QRS amplitude and area are relatively easy to measure and changes in these variables are larger, making them more sensitive to changes in dyssynchrony.

\section{Reliability of Measurements}

Measurements of QRS area by VCG and QRS amplitude by ECG were robust, as indicated by the high value for goodness of sixth order polynomial fitting $\left(R^{2}=0.99\right.$ and $R^{2}=1.00$, respectively). This was considerably better than for $\operatorname{VTI}_{\mathrm{LVOT}}\left(R^{2}=\right.$
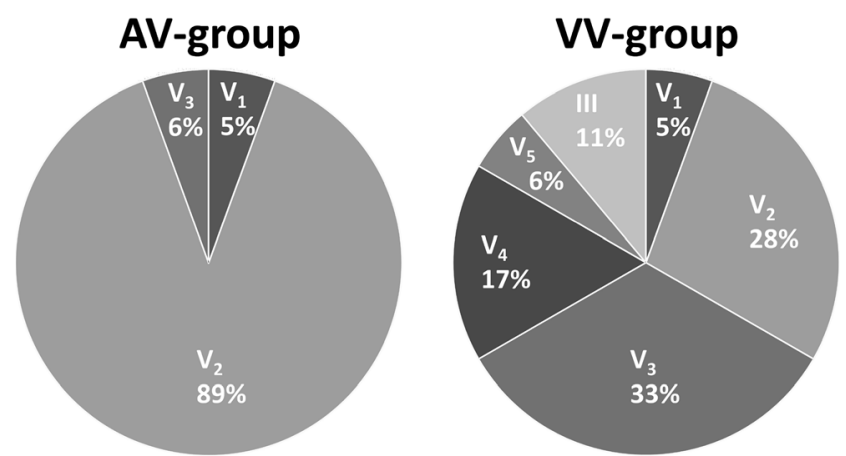

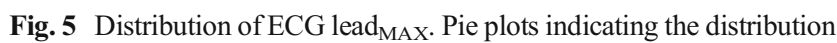
of ECG leads with largest difference in QRS amplitude between LV pacing and LBBB for patients in the AV group (left) and between LV and $\mathrm{RV}$ pacing for patients in the VV group (right) 
0.88), the usual approach for VVoptimization in clinical practice. This relatively low value for VTI was found even despite the use of six averaged beats in our protocol. These findings are in agreement with our previous animal experimental study where vectorcardiographic measurements were highly reproducible with a good signal to noise ratio [15]. The importance of robust and reproducible measurements is highlighted by the search for one specific $\mathrm{AV}$ or $\mathrm{VV}$ interval to select in CRT optimization. This means that the optimum of a curve over different $\mathrm{AV}$ or VV intervals could be at a completely different setting when repeating all $\mathrm{AV}$ or $\mathrm{VV}$ intervals in case of low reproducibility of the measurement (whether because of noise in the measurement or because of physiological influences like loading of the heart, breathing, autonomic nerve system). Avoiding this problem by using a more reproducible measurement as VCG and ECG (less noise, less physiological influence), the optimum can be chosen with confidence. Poor reproducibility of the VTI $\mathrm{LVOT}_{\mathrm{LO}}$ measurement $[9,10]$ may explain why the SMART-AV study was not able to show a benefit using this approach for CRT optimization [5].

\section{Future Implications}

The results from this study indicate that the use of VCG or ECG for $\mathrm{AV}$ and $\mathrm{VV}$ optimization is highly promising. This way of optimization makes it more readily available in all centers and feasible to perform even routinely at follow-up visits, which may be important since remodeling of the heart may change optimal settings over time. The results strongly encourage to perform a larger clinical trial to further support the benefit of ECG and VCG for CRT optimization.

\section{Limitations}

This is a relatively small proof of principle study, which should stimulate to perform a large prospective randomized trial. The small number of patients included may bear the risk of selection of patients. However, Table 1 shows that the patients studied are fairly representative for a CRT population.

The present study only investigated the acute hemodynamic response to CRT. A good acute response does not necessarily imply good long-term outcome [34], so future studies should also address long-term outcome.

We only investigated the use of VCG and ECG for CRT optimization of patients with a right-to-left ventricular activation pattern (LBBB and RV pacing) because such pattern is most amenable to CRT. The results of this study may not apply to right bundle branch block or intraventricular conduction disturbances, but indication for CRT regarding these conduction disturbances is discouraged by current guidelines [35].

While VCG was digitally acquired, ECG measurements were performed manually, which might have been less accurate than using digital calipers. However, by performing them manually, the results of this study can be directly translated in routine clinical practice.

\section{Conclusion}

This study proves the principle that the QRS vector obtained from 3D VCG or from the ECG lead with largest voltage changes is a practical and reliable tool for optimizing stimulation intervals in CRT patients. Larger studies are needed to show whether these measures can be used for routine practice in this field.

Acknowledgments Gunilla Lundahl M.Sc, Gabriel Bergqvist M.Sc, and Lennart Gransberg M.Sc. PhD, who developed the vectorcardiography software sponsored by grants from the Swedish Heart-Lung Foundation and the Sahlgrenska University Hospital, Gothenburg, Sweden.

Conflict of Interest FWP has received research grants from Medtronic Inc, MSD, EBR Systems, and Proteus Biomedical. All other authors state that they have no conflicting interests to declare.

Open Access This article is distributed under the terms of the Creative Commons Attribution License which permits any use, distribution, and reproduction in any medium, provided the original author(s) and the source are credited.

\section{References}

1. Sawhney, N. S., Waggoner, A. D., Garhwal, S., Chawla, M. K., Osborn, J., \& Faddis, M. N. (2004). Randomized prospective trial of atrioventricular delay programming for cardiac resynchronization therapy. Heart Rhythm, 1, 562-567.

2. Whinnett, Z. I., Francis, D. P., Denis, A., Willson, K., Pascale, P., van Geldorp, I., et al. (2013). Comparison of different invasive hemodynamic methods for AV delay optimization in patients with cardiac resynchronization therapy: implications for clinical trial design and clinical practice. International Journal of Cardiology, 168, 2228-37.

3. Perego, G. B., Chianca, R., Facchini, M., Frattola, A., Balla, E., Zucchi, S., et al. (2003). Simultaneous vs. sequential biventricular pacing in dilated cardiomyopathy: an acute hemodynamic study. European Journal of Heart Failure, 5, 305-313.

4. Sogaard, P., Egeblad, H., Pedersen, A. K., Kim, W. Y., Kristensen, B. O., Hansen, P. S., et al. (2002). Sequential versus simultaneous biventricular resynchronization for severe heart failure: evaluation by tissue Doppler imaging. Circulation, 106, 2078-2084.

5. Ellenbogen, K. A., Gold, M. R., Meyer, T. E., Fernndez Lozano, I., Mittal, S., Waggoner, A. D., et al. (2010). Primary results from the SmartDelay determined AV optimization: a comparison to other AV delay methods used in cardiac resynchronization therapy (SMARTAV) trial: a randomized trial comparing empirical, echocardiographyguided, and algorithmic atrioventricular delay programming in cardiac resynchronization therapy. Circulation, 122, 2660-2668.

6. Leon, A. R., Abraham, W. T., Brozena, S., Daubert, J. P., Fisher, W. G., Gurley, J. C., et al. (2005). Cardiac resynchronization with sequential biventricular pacing for the treatment of moderate-to-severe heart failure. Journal of the American College of Cardiology, 46, 2298-2304.

7. Boriani, G., Muller, C. P., Seidl, K. H., Grove, R., Vogt, J., Danschel, W., et al. (2006). Randomized comparison of simultaneous 
biventricular stimulation versus optimized interventricular delay in cardiac resynchronization therapy. The Resynchronization for the HemodYnamic Treatment for Heart Failure Management II implantable cardioverter defibrillator (RHYTHM II ICD) study. American Heart Journal, 151, 1050-1058.

8. Rao, R. K., Kumar, U. N., Schafer, J., Viloria, E., De Lurgio, D., \& Foster, E. (2007). Reduced ventricular volumes and improved systolic function with cardiac resynchronization therapy: a randomized trial comparing simultaneous biventricular pacing, sequential biventricular pacing, and left ventricular pacing. Circulation, 115, 2136-2144.

9. Pabari, P. A., Willson, K., Stegemann, B., van Geldorp, I. E., Kyriacou, A., Moraldo, M., et al. (2011). When is an optimization not an optimization? Evaluation of clinical implications of information content (signal-to-noise ratio) in optimization of cardiac resynchronization therapy, and how to measure and maximize it. Heart Failure Reviews, 16, 277-290.

10. Kyriacou, A., Li Kam Wa, M. E., Pabari, P. A., Unsworth, B., Baruah, R., Willson, K., et al. (2013). A systematic approach to designing reliable VV optimization methodology: assessment of internal validity of echocardiographic, electrocardiographic and haemodynamic optimization of cardiac resynchronization therapy. International Journal of Cardiology, 167, 954-64.

11. Gras, D., Gupta, M. S., Boulogne, E., Guzzo, L., \& Abraham, W. T. (2009). Optimization of AV and VV delays in the real-world CRT patient population: an international survey on current clinical practice. Pacing and Clinical Electrophysiology, 32(Suppl 1), S236-239.

12. Kamdar, R., Frain, E., Warburton, F., Richmond, L., Mullan, V., Berriman, T., et al. (2010). A prospective comparison of echocardiography and device algorithms for atrioventricular and interventricular interval optimization in cardiac resynchronization therapy. Europace, 12, 84-91.

13. Ritter, P., Delnoy, P. P., Padeletti, L., Lunati, M., Naegele, H., BorriBrunetto, A., et al. (2012). A randomized pilot study of optimization of cardiac resynchronization therapy in sinus rhythm patients using a peak endocardial acceleration sensor vs. standard methods. Europace, 14, 1324-1333.

14. Birnie, D., Lemke, B., Aonuma, K., Krum, H., Lee, K. L., Gasparini, M., et al. (2013). Clinical outcomes with synchronized left ventricular pacing: analysis of the adaptive CRT trial. Heart Rhythm, 10, 1368-1374.

15. van Deursen, C. J., Strik, M., Rademakers, L. M., van Hunnik, A., Kuiper, M., Wecke, L., et al. (2012). Vectorcardiography as a tool for easy optimization of cardiac resynchronization therapy in canine left bundle branch block hearts. Circulation. Arrhythmia and Electrophysiology, 5, 544-552.

16. Noble, R. J., Hillis, J. S., Rothbaum, D. A. (1990). Electrocardiology in Walker HK, Hall WD, Hurst JW, eds: Clinical Methods: The History, Physical, and Laboratory Examinations. Butterworths pp 164-185.

17. Auricchio, A., Stellbrink, C., Block, M., Sack, S., Vogt, J., Bakker, P., et al. (1999). Effect of pacing chamber and atrioventricular delay on acute systolic function of paced patients with congestive heart failure. The Pacing Therapies for Congestive Heart Failure Study Group. The Guidant Congestive Heart Failure Research Group. Circulation, 99, 2993-3001.

18. Vernooy, K., Verbeek, X. A., Cornelussen, R. N., Dijkman, B., Crijns, H. J., Arts, T., et al. (2007). Calculation of effective VV interval facilitates optimization of AV delay and VV interval in cardiac resynchronization therapy. Heart Rhythm, 4, 75-82.

19. Arbelo, E., Tolosana, J. M., Trucco, E., Penela, D., Borras, R., Doltra, A., et al. (2014). Fusion-optimized intervals (FOI): a new method to achieve the narrowest QRS for optimization of the AV and VV intervals in patients undergoing cardiac resynchronization therapy. Journal of Cardiovascular Electrophysiology, 25, 283-292.

20. Wecke, L., Rubulis, A., Lundahl, G., Rosen, M. R., \& Bergfeldt, L. (2007). Right ventricular pacing-induced electrophysiological remodeling in the human heart and its relationship to cardiac memory. Heart Rhythm, 4, 1477-1486.
21. van Geldorp, I. E., Delhaas, T., Hermans, B., Vernooy, K., Broers, B., Klimusina, J., et al. (2011). Comparison of a non-invasive arterial pulse contour technique and echo Doppler aorta velocity-time integral on stroke volume changes in optimization of cardiac resynchronization therapy. Europace, 13, 87-95.

22. De Boeck, B. W., Teske, A. J., Meine, M., Leenders, G. E., Cramer, M. J., Prinzen, F. W., et al. (2009). Septal rebound stretch reflects the functional substrate to cardiac resynchronization therapy and predicts volumetric and neurohormonal response. European Journal of Heart Failure, 11, 863-871.

23. Bland, J. M., \& Altman, D. G. (1986). Statistical methods for assessing agreement between two methods of clinical measurement. Lancet, 1, 307-310.

24. Tamborero, D., Vidal, B., Tolosana, J. M., Sitges, M., Berruezo, A., Silva, E., et al. (2013). Electrocardiographic versus echocardiographic optimization of the interventricular pacing delay in patients undergoing cardiac resynchronization therapy. Journal of Cardiovascular Electrophysiology, 22, 1129-1134.

25. Zareba, W., Klein, H., Cygankiewicz, I., Hall, W. J., McNitt, S., Brown, M., et al. (2011). Effectiveness of cardiac resynchronization therapy by QRS morphology in the Multicenter Automatic Defibrillator Implantation Trial-Cardiac Resynchronization Therapy (MADIT-CRT). Circulation, 123, 1061-1072.

26. Jia, P., Ramanathan, C., Ghanem, R. N., Ryu, K., Varma, N., \& Rudy, Y. (2006). Electrocardiographic imaging of cardiac resynchronization therapy in heart failure: observation of variable electrophysiologic responses. Heart Rhythm, 3, 296-310.

27. Ghosh, S., Silva, J. N., Canham, R. M., Bowman, T. M., Zhang, J., Rhee, E. K., et al. (2011). Electrophysiologic substrate and intraventricular left ventricular dyssynchrony in nonischemic heart failure patients undergoing cardiac resynchronization therapy. Heart Rhythm, 8, 692-699.

28. Verbeek, X. A., Auricchio, A., Yu, Y., Ding, J., Pochet, T., Vernooy, K., et al. (2006). Tailoring cardiac resynchronization therapy using interventricular asynchrony. Validation of a simple model. American Journal of Physiology Heart and Circulatory Physiology, 290, H968-977.

29. Strik, M., van Middendorp, L. B., Houthuizen, P., Ploux, S., van Hunnik, A., Kuiper, M., et al. (2013). The interplay of electrical wave fronts as determinant of the response to cardiac resynchronization therapy in dyssynchronous canine hearts. Circulation. Arrhythmia and Electrophysiology, 6, 924-931.

30. Waggoner, A. D., De Las, F. L., Faddis, M. N., Gleva, M. J., Spence, K. E., \& Davila-Roman, V. G. (2008). Left ventricular diastolic filling prior to cardiac resynchronization therapy: implications for atrioventricular delay programming. Pacing and Clinical Electrophysiology, $31,838-844$.

31. Edenbrandt, L., \& Pahlm, O. (1988). Vectorcardiogram synthesized from a 12-lead ECG: superiority of the inverse Dower matrix. Journal of Electrocardiology, 21, 361-367.

32. Kors, J. A., van Herpen, G., Sittig, A. C., \& van Bemmel, J. H. (1990). Reconstruction of the Frank vectorcardiogram from standard electrocardiographic leads: diagnostic comparison of different methods. European Heart Journal, 11, 1083-1092.

33. Varma, N., Jia, P., \& Rudy, Y. (2007). Electrocardiographic imaging of patients with heart failure with left bundle branch block and response to cardiac resynchronization therapy. Journal of Electrocardiology, 40, S174-178.

34. Bogaard, M. D., Houthuizen, P., Bracke, F. A., Doevendans, P. A., Prinzen, F. W., Meine, M., et al. (2011). Baseline left ventricular dP/ dtmax rather than the acute improvement in $\mathrm{dP} / \mathrm{dtmax}$ predicts clinical outcome in patients with cardiac resynchronization therapy. European Journal of Heart Failure, 13, 1126-1132.

35. Prinzen, F. W., Vernooy, K., \& Auricchio, A. (2013). Cardiac resynchronization therapy: state-of-the-art of current applications, guidelines, ongoing trials, and areas of controversy. Circulation, 128, 2407-2418. 